\title{
Erratum to: Extracting kinetic freeze-out temperature and radial flow velocity from an improved Tsallis distribution
}

\author{
Hai-Ling $\mathrm{Lao}^{1}, \mathrm{Fu}-\mathrm{Hu} \mathrm{Liu}^{1, \text { a }}$ and Roy A. Lacey ${ }^{2}$ \\ 1 Institute of Theoretical Physics, Shanxi University, Taiyuan, Shanxi 030006, China \\ 2 Departments of Chemistry \& Physics, Stony Brook University, Stony Brook, NY 11794, USA \\ Original article: Eur. Phys. J. A (2017) 53: 44, DOI: 10.1140/epja/i2017-12238-1 \\ Received: 19 May 2017 \\ Published online: 30 June 2017 - C Società Italiana di Fisica / Springer-Verlag 2017
}

The PHENIX data quoted in this paper should be in the pseudorapidity range $|\eta|<0.35$, but not in the rapidity range $|y|<0.5$; and the ALICE data should be in the range $|\eta|<0.8$ for the high-transverse-momentum region and in the range $|y|<0.5$ for the low-transverse-momentum region, but not only in the range $|y|<0.5$. These corrections do not affect the results of the publication.

The authors regret these mistakes and apologize for any inconvenience caused.

\footnotetext{
${ }^{a}$ e-mail: fuhuliu@163.com
} 